\title{
IRÁN EN AMÉRICA LATINA: PRAGMATISMO Y COMERCIO MÁS ALLÁ DE LOS PAÍSES DEL ALBA*
}

\section{IRAN IN LATIN AMERICA: PRAGMATISM AND TRADE BEYOND THE ALBA COUNTRIES}

\author{
ISABEL RODRÍGUEZ ARANDA**
}

Resumen: Durante el gobierno de Ahmadineyad (2005-2013), Irán desarrolló una relación heterogénea con Latinoamérica, por un lado, una relación política e ideologizada con el eje de países liderados por Venezuela, y por otro, una relación más pragmática con énfasis económico y comercial con otros países de la región. Se analiza en el presente artículo la relación de Irán con Brasil, Uruguay, Argentina, Chile, México, Paraguay y Colombia. Con los tres primeros tiene una relación comercial sostenida en el tiempo y de volumen considerable, con los otros cuatro países, una relación reciente e intensa en lo comercial. Al respecto, la visita del presidente iraní el año 2009 a Brasil marcó un punto de inflexión en este modo pragmático de relación, un factor estratégico en el que los vínculos políticos comenzaron a tener protagonismo fuera del eje ALBA.

Palabras Clave: Irán, América Latina, Brasil, geopolítica.

Abstract: During Mahmoud Ahmadinejad's presidency (2005-2013), Iran developed a diversified relationship with Latin America: on one hand, a political and ideologized relationship with the axis of countries led by Venezuela; and on the other hand, a more pragmatic, economic and business-focused relationship with the other countries of the region. This article analyzes Iran's relationship with Brazil, Uruguay, Argentina, Chile, México, Paraguay, and Colombia. The first three enjoy an historical and substantial business relationship with Iran, while the other countries have established a more recent and intense economic relationship with the Persian country. In this respect, the Iranian president's visit to Brazil in 2009 marked a turning point in this pragmatic relationship by adding a strategic factor in which the political relationship became relevant beyond the ALBA treaty.

KEYwORDs: Iran, Latin America, Brazil, geopolitics.

Recibido: 31.03.17. Aceptado: 15.03.18.

\footnotetext{
* Este artículo es parte del Proyecto de Investigación No 1080024, financiado por el Fondo Nacional de Desarrollo Científico y Tecnológico (FONDECYT) de Chile.

** Doctora en Ciencia Política y Sociología. Investigadora del Centro de Estudios de Relaciones Internacionales (CERI), Facultad de Gobierno, UDD.
} 


\section{INTRODUCCIÓN}

T A DÉCADA DE DOS MIL fue un periodo en que el sistema internacional Lue escenario de importantes cambios en la distribución del poder de potencias tradicionales, emergentes y medianas. El debate neorrealista fue protagonista, donde el consenso mínimo del análisis apuntaba a individualizar un grupo de potencias emergentes que comenzaban un ascenso para alcanzar a la unipotencia, Estados Unidos (Kagan, 2008; Khanna, 2008; Zakaria, 2009). No era el declive de la potencia del norte sino el ascenso de todos los demás.

Entre las potencias medianas de Medio Oriente que van a afectar la estructura de poder global está el caso de Irán, país que desde la revolución islámica de 1979 ha tenido un accionar opuesto a los Estados Unidos y a su unipolaridad, y que en el periodo de análisis de este artículo, 2005-2013, correspondiente al gobierno de Ahmadineyad, mantuvo con este último país relaciones especialmente tensas, principalmente por su programa nuclear y por sus alianzas con países contrarios política e ideológicamente a la unipotencia.

En este marco, llama la atención la relación de Irán con América Latina en este periodo, en cuanto amplía e intensifica sus relaciones con países de la región. Al respecto, desde los análisis periodísticos y también académicos se construyó el mito de que Irán mantenía relaciones únicamente con Venezuela y los países del ALBA; así lo resaltan algunos artículos académicos realizados en el periodo (Caro y Rodríguez, 2009), sin embargo, esta intensa relación política e ideológica con el eje Venezuela fue solo una primera etapa del periodo analizado, porque desde el año 2009 dicho relacionamiento se amplía y refuerza con la visita presidencial iraní a Brasil y la apertura de distintas embajadas, y la intensificación de relaciones comerciales fuera del foco ALBA.

En este artículo analizamos el periodo 2005-2013 para demostrar que el gobierno de Ahmadineyad marca un antes y un después en las relaciones entre Irán y América Latina, en cuanto inaugura una relación económica y política consistente con distintos países más allá del eje de países del ALBA.

Si bien Venezuela va a encabezar un bloque de países de izquierda cuyo propósito es la construcción de una autonomía regional en clara oposición a los Estados Unidos, hay que destacar también a Brasil, que lideró un eje de países con una posición más bien neutral hacia EE.UU., que buscaba la inserción de la región a través de su rol de potencia emergente en el sistema internacional en el marco de su protagonismo en el BRICS en la década ana- 
lizada. Estos países liderados por Brasil y que son objeto de estudio de nuestro análisis son: Uruguay, Argentina, Chile, Paraguay, México y Colombia.

Para fundamentar nuestro planteamiento, este artículo se propone analizar, en términos políticos y económicos, los intereses y la mayor presencia de Irán en este segundo grupo de países para comprobar que Irán, durante el gobierno de Ahmadineyad, desarrolló también una relación fuera de los países del ALBA, con características distintas y apuntando a un pragmatismo estratégico centrado principalmente en lo comercial.

En otras palabras, pese a los cientos de acuerdos que Irán firmó con Venezuela y sus "amigos" en el periodo analizado, el comercio bilateral real que mantuvo con ellos fue relativamente bajo si se lo compara con los otros países mencionados no pertenecientes al ALBA. Al respecto, planteamos que en la política exterior del Presidente iraní desde el 2005 existió un intento serio y explícito de profundizar sus relaciones diplomáticas allí donde las relaciones económicas ya tenían una trayectoria, como es el caso de Uruguay, Argentina y Brasil, y también allí donde estas eran más limitadas pero con potencial comercial, como es el caso de Chile, Paraguay, México y Colombia.

El artículo se estructura en tres partes. La primera analiza el contexto en el que se producen las relaciones entre Irán y América Latina desde el año 2005, haciendo referencia al contexto global en el que Irán intenta salir del aislamiento internacional promovido por EE.UU. En una segunda parte, analizamos la relación de Irán con tres países, Uruguay, Argentina y Brasil, que comparten dos características comunes: tienen una relación bilateral desde hace décadas y han desarrollado consistentes relaciones comerciales con Irán, siendo los mayores socios comerciales de este país en la región. Por último, en una tercera parte, se analizan los países "nuevos" con los que Irán desarrolla relaciones comerciales y de cooperación en este periodo, en específico, Chile, México, Paraguay y Colombia.

\section{IDEOLOGÍA Y PRAGMATISMO EN LA PRESENCIA IRANÍ EN AMÉRICA LATINA}

Durante el gobierno de Ahmadineyad, América Latina se presenta ante los intereses de Irán de manera particular, llegando a transformarse en una de las prioridades de su agenda exterior. Esto se explica, en primer lugar, porque Irán necesita defenderse estratégicamente frente al aislamiento que le impone EE.UU. y sus aliados, en razón de la amenaza que constituye el 
programa nuclear que desarrolla, y en segundo lugar, porque América Latina es un espacio geográfico estratégico que comúnmente se indica como el "patio trasero" de EE.UU. y que el autor Nadereh Farzamnia (2009) resume como "amistades peligrosas cerca de casa" (p. 235).

Esta atención hacia América Latina será relativamente nueva. En efecto, en el anterior gobierno iraní, de Mohamed Jatami (1997-2005), las relaciones con Latinoamérica no variaron sustancialmente. En primer lugar, por lo menos hasta su último año en el mandato, no existió un interés explícito hacia los países latinoamericanos (Jarrazi, 1998, p. 25), y en segundo lugar, porque se intentó seguir una política exterior relativamente pasiva que no dañara aún más las relaciones con EE.UU. (Farhi, 2008). Por lo tanto, fue solo desde que Mahmoud Ahmadineyad resultó electo el año 2005, que comenzó a manifestarse una agenda exterior que le otorga a América Latina una importancia que otrora no tuvo. Y por parte de los países latinoamericanos, si dejamos de lado la importancia estratégica o simbólica que tiene para algunos países, en general Irán se muestra como un país muy atractivo al poseer grandes reservas de petróleo y un mercado de más de 70 millones de personas. En consecuencia, el aspecto político del régimen iraní no será una molestia a la hora de comerciar o establecer alianzas estratégicas.

Es precisamente este interés por Latinoamérica lo que llevó al gobierno iraní a organizar, en el Instituto de Estudios Políticos Internacionales del Ministerio de Asuntos Exteriores, en febrero de 2007, la primera conferencia internacional sobre América Latina, llamada "Desarrollo en América Latina: su papel y su estatus en el futuro sistema internacional", en la que participaron parlamentarios tanto latinoamericanos como de otros países fuera de la región. Cuatro meses más tarde, se realizó el primer "Congreso Internacional de Literatura Latinoamericana", en Teherán e Isfahan, con el propósito de acercar otros ámbitos, además de los políticos y económicos (Malamud y García Encina 2007, p. 2).

Por otra parte, el aspecto diplomático fue otra veta que comenzó a fortalecerse. Así, a las embajadas de Cuba, Venezuela, México, Brasil y Argentina ya existentes, se sumaron las reaperturas, desde el 2007 en adelante, de Uruguay, Nicaragua, Chile, Colombia, Ecuador y Bolivia. De este modo, Irán se transformó en el segundo país del Medio Oriente que tiene la mayor cantidad de personal diplomático en América Latina con nueve embajadas, luego del Líbano con 13 representaciones diplomáticas (Sistema Económico Latinoamericano y del Caribe, 2011).

En este contexto, es importante destacar para los objetivos de este artículo, que la estrategia de inserción iraní en Latinoamérica ha tenido dis- 
tintas intenciones y motivaciones, dependiendo de los países de la región. Existe un primer grupo de países, los pertenecientes al ALBA, donde destaca el factor ideológico-político, que también incluye acuerdos comerciales y culturales. En este sentido, una de las razones fundamentales de por qué el Presidente iraní intensificó desde un comienzo sus lazos con Venezuela y sus "amigos", fue la de defenderse y ganar apoyo frente a las presiones y aislamiento por parte de EE.UU. y los países europeos, siendo su puerta de entrada al continente la profundización de sus relaciones con la Venezuela de Chávez. Ambos mandatarios se encontraron en un objetivo común: su rechazo y crítica al gobierno de Washington. De este modo, Irán creó con sus vecinos regionales una alianza musulmana anti-Estados Unidos, entre ellos Siria, Hamas en Palestina y grupos chiítas de Irak y el Líbano, mientras que Venezuela ha hecho lo suyo en América Latina con Cuba, Bolivia, Nicaragua, Ecuador y República Dominicana (Caro y Rodríguez, 2009, p. 28). Aquí se produce, entonces, una convergencia de intereses que incorpora a América Latina como parte del escenario de esta geopolítica global.

Esta aproximación entre los países pertenecientes al ALBA e Irán se ha traducido en que los primeros hayan hecho público o hayan manifestado respeto ante el programa de energía nuclear del país persa (Caro y Rodríguez, 2009, p. 34), al tiempo que todos ellos han realizado esfuerzos políticos para, en cada caso, profundizar y restablecer lazos con Irán en un intento de diversificar sus lazos económicos y encontrar apoyo político en contra del "imperialista país del norte".

Ahora bien, más allá de estos importantes vínculos que se han traducido en viajes oficiales, inversiones, relaciones comerciales y un sinfín de acuerdos de todo tipo, existe otro grupo de países que, de manera menos estridente y sin tanta parafernalia publicitaria, fue captando la atención iraní de manera importante en el gobierno de Ahmadineyad, configurándose como un objetivo en materia de su política exterior, despejando el mito de que la relación de Irán con la región en el periodo señalado está centrada únicamente en los países del ALBA por el componente político e ideológico.

El punto de inflexión ciertamente fue la visita del presidente iraní a Brasil en noviembre de 2009, con lo cual la relación estratégica de Irán con América Latina incluye el factor político-estratégico más allá del eje de países del ALBA. Al respecto, planteamos que, en la política exterior del ex presidente iraní, ha existido un intento serio y explícito de profundizar sus relaciones diplomáticas allí donde las relaciones económicas habían sido muy prósperas, o allí donde estas eran más limitadas pero de igual modo potencialmente favorables. Para ello, analizaremos Brasil, Argentina, Uru- 
guay, Paraguay, Colombia, Chile y México, desde la asunción del presidente Ahmadineyad.

En otras palabras, pese a los cientos de acuerdos que Irán ha firmado con Venezuela y sus "amigos", el comercio bilateral real que mantiene con ellos es relativamente bajo si se lo compara con otros países no pertenecientes al ALBA; por ejemplo, con Venezuela, entre los años 2005 y 2010, Irán tuvo un comercio que promedió US\$ 47,4 millones (Hinds, 2011), siendo similar al que tiene con México y muy por debajo del que mantenía con Uruguay, Argentina, Chile y, por sobre todo, con Brasil (Botta, 2009). Este intercambio comercial con Venezuela disminuyó a US\$36,2 millones en 2013 y cesó por completo en 2014, año desde el cual se ha mantenido de esa manera, mientras que con los demás países, si bien el comercio ha disminuido, se mantiene (Center for International Development at Harvard University, 2018). Por lo tanto, como se analizará en los próximos apartados, la penetración comercial y diplomática de Irán en América Latina más allá de los países del ALBA está reflejando una veta poco examinada, mostrando que es una coyuntura donde Irán integra una diplomacia basada en el comercio y la cooperación, con una diplomacia ideologizada que se enmarca en su juego geopolítico global.

\section{IRÁN Y SUS MAYORES SOCIOS COMERCIALES: URUGUAY, ARGENTINA Y BRASIL}

Los países que analizaremos a continuación tienen en común el tener relaciones diplomáticas o formales con Irán desde hace décadas: Uruguay desde 1976, Argentina desde 1902 y Brasil desde 1903, siendo Irán el primer Estado del Medio Oriente con el que establecen relaciones diplomáticas estos últimos países. También tienen en común el haber desarrollado una permanente e intensa relación comercial, que según cifras que especificaremos en el análisis, resulta mayor en una comparación uno a uno, a todo el resto de los países de la región, incluyendo a los países que conforman el ALBA.

\subsection{Uruguay}

Analizaremos en primer lugar el caso de Uruguay, que es tal vez uno de los países latinoamericanos que ha mantenido una de las relaciones más estables con la República de Irán, la que se ha basado, fundamentalmente, 
en un intenso y complementario vínculo comercial recíproco. Este estable vínculo comercial se ha sustentado en la abstención por parte de Uruguay a las condenas que ha llevado a cabo la Asamblea General de Naciones Unidas a la situación de los derechos humanos en Irán desde 1976.

Las relaciones diplomáticas entre ambos países comenzaron precisamente en 1976, y desde 1984 en adelante, han existido misiones permanentes. El rango de sus delegaciones es el de embajada, lo que según Pablo Brum y Mariana Dambolena (2007, p. 3), tiene un significado principalmente económico, es decir, poder garantizar los intercambios comerciales privados. En efecto, desde fines de la década de 1970, Irán ha exportado petróleo a Uruguay, mientras que los empresarios uruguayos han colocado en el mercado iraní productos como pollo, carne y arroz. Ha sido, sobre todo este último producto, el que más ha importado Irán, el cual tuvo un enorme crecimiento cuando, tras los atentados terroristas a la AMIA y la acusación argentina al país persa, Irán decidió solo comprar arroz a Uruguay. Lo que no fue menor, si consideramos que la cifra de exportación de arroz argentino ascendía a US\$ 900 millones, tomando como referencia el año 1994 (Brum y Dambolena, 2007, p. 4).

Algunas cifras relevantes en términos de intercambio comercial son: hacia el año 2005, las exportaciones uruguayas fueron superiores a los US $\$ 80$ millones, mientras que para los años 2004 y 2006, fueron US\$ 52 millones y US\$ 42 millones respectivamente; las ventas del principal producto de exportación, es decir el arroz, tuvo hacia el 2005 una cifra récord de US\$ 74 millones, mientras que para el año 2008 fue de alrededor de US\$ 70 millones; las cifras de importación de petróleo iraní fueron de US\$ 70 millones para el 2006 y US\$ 60 millones para el 2007 (La Red 21, 2008). El intercambio comercial entre ambos países alcanza su punto máximo en 2008 con US\$125,9 millones, y disminuye a US\$ 78,6 millones en 2011. A pesar de que esta cifra siguió disminuyendo, alcanzando US\$ 72,2 millones en el 2013, el intercambio comercial entre ambos países continúa hasta la actualidad (Center for International Development at Harvard University, 2018). Como señaló el embajador uruguayo en Irán (La Red 21, 2008), un aspecto del intercambio comercial que ha sido fundamental es la estabilidad y la confianza que históricamente ha existido entre ambos países.

\subsection{Argentina}

A este país lo une con Irán una historia diplomática de casi 60 años, junto a una historia de intercambio comercial desde hace varias décadas. Hasta por 
lo menos 1992, cuando ocurre el primero de los dos atentados terroristas, los vínculos comerciales eran muy estrechos, pues al igual que Uruguay, Argentina exportaba productos agrícolas de consumo básico mientras que importaba petróleo de Irán. Así, el comercio bilateral, hacia fines de 1980, ascendía a los US\$ 2.000 millones anuales, siendo junto con Brasil el país latinoamericano que tenía el comercio más importante con el país persa (Diario Los Andes, 2007). Asimismo, existía cooperación en el ámbito de la tecnología nuclear, concretándose acuerdos entre una de las empresas del Estado argentino, el INVAP, y la organización de energía atómica de Irán (Jaim, 2009). Estos estrechos vínculos se tradujeron, al igual que en el caso de Uruguay, en que Argentina también se abstuviera de condenar a Irán por las acusaciones contra los derechos humanos ante la Asamblea General de las Naciones Unidas y, cuando lo hizo, fue en 1993 y 1994, años de plena tensión en las relaciones (Brum y Dambolena, 2007, p. 3).

No obstante lo anterior, con Argentina, la situación es diferente a los demás países de la región; mientras Irán se hace cada vez más presente y fortifica sus vínculos económicos y políticos con Latinoamérica, las relaciones con el gobierno de Cristina Fernández experimentaron un estancamiento en los últimos años del periodo analizado. Este deterioro se explica a causa de dos atentados terroristas ocurridos en Argentina, uno el año 1992 y el otro en 1994. El primero, cuando se detonó un vehículo con explosivos en la embajada de Israel en Buenos Aires, dejó 29 muertos. El segundo, cuando nuevamente se detonó un vehículo con explosivos en la Asociación Mutual Israelita Argentina (AMIA), el mayor centro de la comunidad judía que existe en América Latina, asesinando a 85 personas (Brum y Dambolena, 2007, p. 3).

Tras estos atentados, se sospechó en las distintas investigaciones que estuvo la mano del gobierno iraní de entonces. Esto se tradujo en un quiebre en las relaciones políticas, aunque sin cerrar las respectivas embajadas y, manteniéndose, aunque no sin tensión, los vínculos comerciales. En este sentido, históricamente las relaciones entre ambos países se tienen que leer marcadas por un antes y un después de lo ocurrido en la primera mitad de los noventa.

Pese a las tensionadas relaciones entre ambos países desde mediados de la década de 1990, los vínculos comerciales nunca dejaron de ser importantes: las exportaciones argentinas a Irán, durante 2007, fueron de US\$ 319,3 millones, y el principal producto fue el aceite de soja en bruto con US\$ 167,5 millones (Campuzano, Dueñas, Lamar y Rodríguez, 2008, p. 5), mientras que el comercio total entre ambos países, durante 2010, fue calcu- 
lado en US\$ 1476,7 millones. Nuevamente, existe una disminución en esta cifra, llegando a US\$ 988,4 millones en 2012 y a US\$ 1.103,1 millones en 2013 (Center for International Development at Harvard University, 2018).

\subsection{Brasil}

Brasil es, sin duda, el país latinoamericano que más comercia con Irán, lo cual es ampliamente favorable para el primero. En el periodo analizado, Brasil era una potencia emergente, miembro del BRICS, y con un importante crecimiento de su economía, por lo tanto, para Irán era de mucho interés un mayor acercamiento, para poder mostrar ante la comunidad internacional y al interior de su propio país, que se es "amigo" de una potencia emergente que tiene estrechas relaciones tanto con EE.UU. y Europa, como con China, India y Rusia. Es decir, el gobierno iraní, al acercarse a Brasil, sabía que ganaba en prestigio y que podía obtener, cuando fuera necesario, el favor de un "grande" del juego internacional.

Si bien los estrechos vínculos comerciales han estado presentes desde hace mucho tiempo, no fue sino durante 2004, en la Cumbre del G-15 en Caracas, donde reunido Mohammed Jatami con Lula da Silva de Brasil, que estos comenzaron a profundizarse. Ambos mandatarios firmaron un Memorando de Entendimiento para consolidar los intercambios comerciales, así como para cooperar en varias áreas, como en la textil y la automotriz. Asimismo, en el área de la energía, Petrobras firmó un contrato con la $\mathrm{Na}$ tional Iranian Oil Company (NIOC) que tenía el objetivo de explorar el bloque Tusan. De esta manera, como veremos, las relaciones diplomáticas y comerciales entre ambos países se fortalecieron desde entonces (Malamud y García Encina, 2007, p. 5), e incluso, en solo un par de años, se duplicaron (Farhi, 2008).

Algunos datos a este respecto, son que el comercio en el año 2013 entre ambos países superó los dos billones de dólares anuales; y el mercado bilateral, en ese mismo año, fue casi cuatro veces superior al de 2002. Sin embargo, los beneficios no son recíprocos, pues las exportaciones iraníes a Brasil apenas superan los 70 millones de dólares. Este intercambio comercial con Brasil representa el $81 \%$ de las relaciones que tiene Irán con América Latina, mientras que Argentina ocupa el segundo lugar con un 15,5\% (Center for International Development at Harvard University, 2018).

Por último, es interesante señalar que estas estrechas relaciones comerciales y diplomáticas han tenido efectos en el plano político. Un ejemplo de ello fue cuando Brasil, en noviembre de 2007, se abstuvo de votar en el 
plenario de la INTERPOL para que se analizara el pedido de Argentina, apoyada por EE.UU., para pedir la captura internacional de una decena de iraníes acusados de los atentados contra la AMIA. El argumento expuesto por el gobierno brasileño fue del todo pragmático y ligado a sus intereses nacionales, explicando que, para él, era fundamental cuidar la creciente relación económica y energética que mantenía con Irán (Calle, 2009).

Además, la visita de Ahmadineyad en noviembre de 2009 a Brasil fue un punto de inflexión en las relaciones entre ambos países, pero también un aspecto relevante para la relación estratégica que Irán podía impulsar en el mediano plazo con los países de la región alejados del eje ALBA. Para Ahmadineyad era conveniente realizar esta visita antes de las elecciones presidenciales de su país, ya que ayudaba a fortalecer su estrategia contra el aislamiento internacional promovido por EE.UU. y sus aliados. Un segundo dato interesante es que luego de las elecciones presidenciales, Lula da Silva fue uno de los primeros mandatarios en cuestionar las denuncias de fraude en la reelección de Ahmadineyad. Asimismo, y como tercer aspecto, Brasil se ha mostrado proclive a la posibilidad de desarrollar tecnología nuclear con fines pacíficos y, por lo tanto, hay un espacio ganado para desarrollar una amplia cooperación con Irán en este aspecto tan controversial en las relaciones de poder de las grandes potencias.

\section{LA AMPLIACIÓN DE RELACIONES DE IRÁN CON CHILE, MEXICO, PARAGUAY Y COLOMBIA}

La presencia de Irán en estos países no ha sido similar en el tiempo ni en la profundidad a la del grupo de países ya analizados, pero tienen el común denominador de ser relaciones relativamente nuevas o que van en ascenso en los últimos años. El primer país que analizaremos es Chile, con el que Irán ha intensificado sus relaciones recién desde el año 2007. En términos históricos, las relaciones diplomáticas entre ambos países se inician en 1991, tras el regreso de Chile a la democracia.

En el aspecto económico, Chile es un importante socio comercial de Irán. Hacia el año 2007, las ventas externas al país persa eran de alrededor de US\$ 181,6 millones, de los cuales los minerales de cobre y sus concentrados fueron el principal producto de exportación con US\$ 176,3 millones (Campuzano et al., 2008, p. 5). Entre los años 2010 y 2013 pasan a ser los artículos de hierro y acero, junto con las fibras de lana naturales, los principales productos exportados, llegando a ser un $82 \%$ de las exportaciones 
con US\$ 3,33 millones (Center for International Development at Harvard University, 2018).

México es otro de los países que ha tenido, en el último tiempo, un importante acercamiento con Irán. Si bien las relaciones diplomáticas se iniciaron hacia 1964, manteniéndose dentro de un plano de conveniencia mutua comercial, y con incipientes relaciones económicas y tecnológicas, no fue sino hasta el gobierno de Ahmadineyad cuando estas comenzaron a profundizarse. En efecto, estas experimentaron un nuevo vuelco en el 2004, año en que el Presidente mexicano, Vicente Fox, recibió la visita del entonces Ministro de Relaciones Exteriores de Irán, Kaml Kharrazi. En el encuentro se desarrollaron iniciativas para fortalecer las relaciones entre empresarios, así como realizar consultas de concertación económica y política. Importante también es señalar que se suscribió el Programa de Cooperación Educativo y Cultural para el período 2004-06 (Cobos González, 2004).

Sin embargo, tuvieron que transcurrir cuatro años para que nuevamente se expresara esta voluntad de intensificar las relaciones entre estos dos países. Fue así como en febrero de 2009, el Vice Ministro de Relaciones Exteriores de Irán para las Américas, Alí Reza Salari, viajó a México en un intento por ampliar los vínculos en los ámbitos político, cultural y, por sobre todo, económico. En este sentido, un objetivo prioritario de la visita fue el poder aumentar el intercambio comercial, que en ese entonces estaba alrededor de los US\$50 millones de dólares anuales (Botta, 2009). Esta cifra disminuye para el 2013, alcanzando los US\$20,9 millones (Center for International Development at Harvard University, 2018).

En el caso de Paraguay, de manera similar a Chile, las relaciones comenzaron a principios de la década de 1990, manteniéndose débiles o casi inexistentes por un largo período de tiempo. Sin embargo, con el triunfo presidencial de Fernando Lugo en agosto de 2008, los vínculos dejaron la simple formalidad. Y esto fue así, porque la llegada al poder de Lugo fue considerada por Irán como una potencial aliada para contrapesar la influencia de los Estados Unidos en el continente, principalmente, porque la victoria de Lugo vino a terminar con más de 70 años de predominio conservador y porque Lugo siempre planteó en su programa de gobierno resistir la influencia de Washington. Esto llevó a un aumento progresivo en el intercambio comercial entre Irán y Paraguay, el que en 2013 representó US\$ 7 millones (Center for International Development at Harvard University, 2018).

Por último, Colombia es uno de los países grandes de la región donde 
Irán tiene una muy baja presencia económica. Sin embargo, esta fue en aumento en el periodo analizado. Las exportaciones colombianas, históricamente, no han representado más que el $1 \%$ del total vendido, y del cual el banano ha sido el principal producto de exportación; durante 2007, las ventas a Irán fueron de US\$140.000 (Campuzano et al., 2008, p. 4), lo que es muy bajo en comparación con los otros países latinoamericanos analizados. Por el contrario, las importaciones de productos iraníes a Colombia han sido por un valor mucho más alto: entre 2003 y 2006, el valor de las importaciones fue de US\$ 470.000 en promedio anual; pero durante 2007, estas se incrementaron en un 405\%, totalizando un valor de US\$ 2,8 millones, que se explica por el gran aumento de la compra de semillas de comino (Campuzano et al., 2008, p. 11). Esta situación se mantiene hasta el 2013, año en el que Colombia importó desde Irán US\$1,19 millones, y exportó a este país US\$ 50.000 (Center for International Development at Harvard University, 2018).

\section{CONCLUSIONES}

Analizamos los nexos entre Irán y Latinoamérica durante el gobierno de Ahmadineyad. Sin duda que el mayor esfuerzo político y diplomático se lo han llevado Venezuela y los países que están liderados por él, como Ecuador, Nicaragua y Bolivia, en un intento de crear una alianza estratégica e ideológica que apoye su programa de energía nuclear y se oponga a EE.UU. En este contexto, constatamos en este artículo que en el periodo 2005-2013 ha existido por parte de Irán un esfuerzo por ampliar sus relaciones diplomáticas y económicas con otros países que no comparten con él ningún tipo de afinidad política o ideológica, como son Brasil, Uruguay, Argentina, Chile, México, Paraguay y Colombia, por lo que la presencia de Irán ha tenido un objetivo conjunto en la región, sin privilegiar a aquellos países con relaciones de larga data o a aquellos dentro del ALBA. Debido a la ubicación geográfica de América Latina, lo anterior es clave para Irán, pues está consolidando su presencia en países que históricamente han sido zona de influencia norteamericana.

Al respecto y luego de hacer un análisis individual por país, vemos que los tres primeros han tenido una relación comercial sostenida en el tiempo con Irán y de volumen considerable, mientras que los otros tres han establecido desde el 2005 prósperas relaciones comerciales. En general, Irán resulta un socio atractivo por sus abundantes reservas de petróleo y porque 
posee un mercado aproximado de 70 millones de personas; por lo tanto, los países analizados han visto a Irán como un país más con el cual dialogar y comerciar.

En consecuencia, son estas nuevas relaciones analizadas, más allá de Venezuela y sus "amigos", lo que nos permite concluir que con el gobierno de Ahmadineyad se inició por parte de Irán una política exterior más pragmática en torno a abrirse a establecer, o restablecer, vínculos allí donde antes habían sido limitados o eran puramente económicos. Comprueba lo anterior, los viajes de altos funcionarios iraníes a México y Brasil, la reapertura o apertura de embajadas, las nuevas relaciones con un Paraguay que, si bien políticamente de izquierda, no es parte del grupo de países que sigue al Presidente venezolano, y lo mismo ocurre con el gobierno electo de Uruguay en este periodo. En el plano económico, destacan los estrechos vínculos con Brasil y Uruguay, mientras fortalece las relaciones comerciales con Chile, México y Paraguay.

En esta misma línea, hechos como la visita del presidente iraní en noviembre de 2009 a Brasil demostraron que efectivamente Irán sumó al pragmatismo de las relaciones con los países fuera del eje ALBA un aspecto político-estratégico. Convergen aquí intereses de ambas potencias emergentes del sistema internacional. Por parte de Irán, destaca su interés de salir del aislamiento internacional, de construir más presencia en América Latina y lograr acercamiento a países aliados de EE.UU. A su vez, por parte de Brasil, su interés estaba en jugar un rol más destacado en el sistema internacional, tanto como potencia económica como también en el plano político, demostrando tener una diplomacia eficiente para atender los conflictos internacionales. Todo lo anterior nos permite constatar el nuevo rumbo que tomaba la agenda exterior de Irán en América Latina en el periodo analizado.

\section{REFERENCIAS}

Botta, P. (5 de mayo de 2009). Ahmadineyad en América Latina. Recuperado de Información y Análisis de América Latina: http://www.infolatam. com/2009/05/03/ahmadineyad-en-america-latina/

Brum, P. y Dambolena, M. (10 de diciembre de 2007). Irán y el Río de la Plata: Las relaciones de la República Islámica con Argentina y Uruguay. Recuperado de CADAL: http://www.cadal.org/documentos/nota.asp?id_nota=2207

Calle, F. (9 de febrero de 2009). La estrategia de inserción de Irán en América Latina en el siglo XXI. Recuperado de Nueva Mayoría: http://www.nue- 
vamayoria.com/index.php?option=com_content\&task=view\&id=1213\&It emid $=38$

Campuzano, C., Dueñas, L., Lamar, J. y Rodríguez, T. (2008, octubre). Informe de Exportaciones, Turismo e Inversión Extranjera. Recuperado de Proexport Colombia: http://antiguo.proexport.com.co/vbecontent/library/documents/DocNewsNo10050DocumentNo7842.pdf

Caro, I. y Rodríguez, I. (2009). La presencia de Irán en América Latina a través de su influencia en los países del Alba. Atenea, 500, 21-39.

Center for International Development at Harvard University (2018). The Atlas of Economic Complexity. Recuperado de: http://atlas.cid.harvard.edu/

Cobos González, C. (2004, junio 30). Recibe el Presidente Vicente Fox al Ministro de Relaciones Exteriores de Irán, Kamal Kharrazi. Recuperado de Presidencia de la República, Vicente Fox: http://fox.presidencia.gob.mx/ actividades $/$ ?contenido $=8512$

Diario Los Andes (2007, marzo 3). D’Elía trajo un mensaje conciliador de Irán. Recuperado de Diario Los Andes: http://www.losandes.com.ar/notas/2007/3/3/politica-222706.asp

Farhi, F. (2008, julio 10). Conference: Iran in LatinAmerica: Threator Axis of Annoyance. Recuperado de Woodrow Wilson International Center for Scholars: https://www.wilsoncenter.org/sites/default/files/Farhi.pdf

Farzamnia, N. (2009). Irán: de la revolución islámica a la revolución nuclear. Madrid, España: Editorial Síntesis.

Hinds, A. (2011, mayo 27). INTERCAMBIO: Comercio con Irán aumentó $131 \%$ en el primer trimestre. Recuperado de Reportero 24: http://www. reportero24.com/2011/05/intercambio-comercio-con-iran-aumento131-en-el-primer-trimestre/

Jaim, B. (2009, agosto 10). Irán. ¿Qué futuro se avecina? Recuperado de La Voz Judía: http://www.delacole.com/cgi-perl/medios/vernota.cgi?medio=lavoz judia\&numero=371\&nota $=371-12$

Jarrazi, K. (1998). “Nuevas perspectivas en la política exterior de Irán”. Política Exterior, 12, 23-29.

Kagan, R. (2008). El retorno de la historia y el fin de los sueños. Madrid, España: Editorial Taurus.

Khanna, P. (2008). El Segundo Mundo: imperios e influencia en el nuevo orden mundial. Barcelona, España: Editorial Paidós.

La Red 21. (2008, julio 21). Uruguay busca incrementar su relación comercial con Irán. Recuperado de La Red 21: http://www.larepublica.com.uy/ economia/320940-uruguay-busca-incrementar-su-relacion-comercialcon-iran

Malamud, C. y García Encina, C. (2007). "Los actores extrarregionales en América Latina (II): Irán”. Boletín del Real Instituto Elcano, 97, 22-27.

Sistema Económico Latinoamericano y del Caribe (marzo de 2011). Las relaciones de América Latina y el Caribe con el Medio Oriente: Situación actual 
y áreas de oportunidad. Recuperado de AGCI Ministerio de Relaciones Exteriores del Gobierno de Chile: http://www.agci.cl/attachments/article/695/ T023600004688-0-Relaciones_de_ALC_con_el_Medio_Oriente.pdf

Zakaria, F. (2009). El mundo después de USA. Madrid, España: Editorial Espasa Calpe. 